\author{
M.T. Kosmakova ${ }^{1}$, V.G. Romanovski ${ }^{2}$, N.T. Orumbayeva ${ }^{1}$, \\ Zh.M. Tuleutaeva ${ }^{1}$, L.Zh. Kasymova $^{1}$ \\ ${ }^{1}$ Buketov Karaganda State University, Kazakhstan; \\ ${ }^{2}$ University of Maribor, Slovenia \\ (E-mail: Svetik_mir69@mail.ru)
}

\title{
On the integral equation of an adjoint boundary value problem of heat conduction
}

\begin{abstract}
An integral equation is considered, to which a nonhomogeneous first boundary value problem with an adjoint heat conduction operator is reduced. The problem is set in an infinite plane angle, that is, a boundary of the domain moves with a constant velocity, and the domain degenerates to a point at the initial moment of time. The incompressibility of the integral operator for the equation under study is shown. Using the relations for an independent variable, the equation under study is equivalently reduced to a certain simplified equation. With the help of replacements for independent variables, the equation is reduced to an integral equation with a difference kernel. By applying the Laplace transform, the obtained equation is reduced to an ordinary first-order differential equation (linear). Its solution is found. By using the inverse Laplace transform, a solution of the nonhomogeneous integral equation under study is obtained in the form of a convergent series in some domain.
\end{abstract}

Keywords: heat conduction, nonhomogeneous singular integral equation, adjoint boundary value problem, Laplace transform.

\section{Introduction}

In the study of some nonlocal internal-boundary problems for a parabolic equation, spectrally loaded parabolic equations, problems with a moving boundary and inverse problems for parabolic equations, etc. there is a need to study singular integral equations of the form:

$$
\begin{gathered}
\psi(t)-\frac{1}{2 a \sqrt{\pi}} \int_{t}^{\infty}\left[\frac{\tau+t}{(\tau-t)^{\frac{3}{2}}} \exp \left\{-\frac{(\tau+t)^{2}}{4 a^{2}(\tau-t)}\right\}+\right. \\
\left.+\frac{1}{(\tau-t)^{\frac{1}{2}}} \exp \left\{-\frac{\tau-t}{4 a^{2}}\right\}\right] \psi(\tau) d \tau=f(t), \quad(t>0) .
\end{gathered}
$$

The boundary value problems in the case of temperature heating are reduced to such equations (the first boundary value problem):

with boundary conditions:

$$
\frac{\partial v}{\partial t}=-a^{2} \frac{\partial^{2} v}{\partial x^{2}}
$$

$$
\left.v(x, t)\right|_{x=0}=v^{*}(t),\left.\quad v(x, t)\right|_{x=t}=\omega^{*}(t),\left.\quad v(x, t)\right|_{t=\infty}=0 .
$$

1. Incompressibility of an integral operator and reducing

the integral equation to an equation with a difference kernel

For the kernel of equation (1):

$$
K(\tau, t)=\frac{1}{2 a \sqrt{\pi}}\left[\frac{\tau+t}{(\tau-t)^{\frac{3}{2}}} \exp \left\{-\frac{(\tau+t)^{2}}{4 a^{2}(\tau-t)}\right\}+\frac{1}{(\tau-t)^{\frac{1}{2}}} \exp \left\{-\frac{\tau-t}{4 a^{2}}\right\}\right]
$$


we have:

$$
\lim _{t \rightarrow \infty} \int_{t}^{\infty} K(\tau, t) d \tau=\lim _{t \rightarrow \infty}\left(2 e^{-\frac{2 t}{a^{2}}}+1\right)=1_{+0} .
$$

Hence, the characteristic part of equation (1) is the second term of the kernel (2).

Using relations:

$$
\tau+t=2 \tau-(\tau-t), \quad \frac{(\tau+t)^{2}}{4 a^{2}(\tau-t)}=\frac{\tau t}{a^{2}(\tau-t)}+\frac{\tau-t}{4 a^{2}}
$$

equation (1) will be rewritten as:

$$
\begin{gathered}
\psi(t)-\int_{t}^{\infty} \frac{1}{2 a \sqrt{\pi}}\left\{\frac{2 \tau}{(\tau-t)^{3 / 2}} \exp \left\{-\frac{\tau t}{a^{2}(\tau-t)}\right\}+\right. \\
\left.+\frac{1}{\sqrt{\tau-t}}\left(1-\exp \left\{-\frac{\tau t}{a^{2}(\tau-t)}\right\}\right)\right\} \cdot \exp \left\{-\frac{\tau-t}{4 a^{2}}\right\} \psi(\tau) d \tau=f(t) .
\end{gathered}
$$

It is enough to find a solution to the «simplified» equation:

$$
\psi(t)-\int_{t}^{\infty} k^{*}(t, \tau) \psi(\tau) d \tau=g(t)
$$

where

$$
\begin{gathered}
k^{*}(t, \tau)=\frac{1}{2 a \sqrt{\pi}}\left\{\frac{2 \tau}{(\tau-t)^{3 / 2}} \exp \left\{-\frac{\tau t}{a^{2}(\tau-t)}\right\}+\frac{1}{\sqrt{\tau-t}}\left(1-\exp \left\{-\frac{\tau t}{a^{2}(\tau-t)}\right\}\right)\right\} \\
g(t)=\exp \left\{-\frac{t}{4 a^{2}}\right\} \cdot f(t) .
\end{gathered}
$$

We consider the integral equation (3):

$$
\begin{aligned}
& \psi(t)-\frac{1}{2 a \sqrt{\pi}} \int_{t}^{\infty}\left\{\frac{2 \tau}{(\tau-t)^{3 / 2}} \exp \left\{-\frac{\tau t}{a^{2}(\tau-t)}\right\}+\right. \\
& \left.+\frac{1}{\sqrt{\tau-t}}\left(1-\exp \left\{-\frac{\tau t}{a^{2}(\tau-t)}\right\}\right)\right\} \psi(\tau) d \tau=g(t) .
\end{aligned}
$$

Integral equation (4) is reduced to an equation with a difference kernel by means of replacements:

$$
t=\frac{1}{t_{1}}, \quad \tau=\frac{1}{\tau_{1}}
$$

and notation:

$$
y\left(t_{1}\right)=\frac{1}{t_{1}^{3 / 2}} \cdot \psi\left(\frac{1}{t_{1}}\right), \quad g_{1}\left(t_{1}\right)=\frac{1}{t_{1}^{1 / 2}} \cdot g\left(\frac{1}{t_{1}}\right) .
$$

As a result, we obtain the equation:

$$
\begin{gathered}
t_{1} \cdot y_{1}\left(t_{1}\right)-\frac{1}{2 a \sqrt{\pi}} \int_{0}^{t_{1}} \frac{1}{\left(t_{1}-\tau_{1}\right)^{1 / 2}}\left(1-\exp \left\{-\frac{1}{a^{2}\left(t_{1}-\tau_{1}\right)}\right\}\right) y\left(\tau_{1}\right) d \tau_{1}- \\
-t_{1} \cdot \frac{1}{2 a \sqrt{\pi}} \int_{0}^{t_{1}} \frac{2}{\left(t_{1}-\tau_{1}\right)^{3 / 2}} \exp \left\{-\frac{1}{a^{2}\left(t_{1}-\tau_{1}\right)}\right\} y\left(\tau_{1}\right) d \tau_{1}=g_{1}\left(t_{1}\right)
\end{gathered}
$$




\section{Solution of a homogeneous equation with a difference kernel}

Applying the Laplace transform to the equation (5) we obtain the operator equation:

$$
-\bar{y}^{\prime}(p)-\frac{1}{2 a \sqrt{p}}\left(1-\exp \left(-\frac{2 \sqrt{p}}{a}\right)\right) \bar{y}(p)+\left\{\exp \left(-\frac{2 \sqrt{p}}{a}\right) \bar{y}(p)\right\}^{\prime}{ }^{\prime}=\bar{G}_{1}(p) .
$$

After simple transformations we finally get:

$$
\bar{y}^{\prime}(p)+\frac{1}{2 a \sqrt{p}} \frac{\operatorname{ch} \frac{\sqrt{p}}{a}}{\operatorname{sh} \frac{\sqrt{p}}{a}} \bar{y}(p)=-\frac{\bar{G}_{1}(p)}{1-\exp \left(-\frac{2 \sqrt{p}}{a}\right)} .
$$

The solution of the differential equation (6) is the following function:

$$
\bar{y}(p)=\frac{C}{\operatorname{sh} \frac{\sqrt{p}}{a}}-\frac{1}{2 \operatorname{sh} \frac{\sqrt{p}}{a}} \int_{p}^{\infty} \bar{G}_{1}(q) \exp \left(\frac{\sqrt{q}}{a}\right) d q .
$$

The solution of the homogeneous equation corresponding to (6) is the following function:

$$
\bar{y}_{h o m}(p)=\frac{C}{\operatorname{sh} \frac{\sqrt{p}}{a}} .
$$

(1-st term on the right side of the expression (7)).

To (8) we apply the inverse Laplace transform:

$$
y_{h o m}\left(t_{1}\right)=-C\left[\frac{\partial}{\partial \nu} \widehat{\theta}_{0}\left(\frac{\nu}{2} ; a^{2} t_{1}\right)\right]_{\nu=0}
$$

where

$$
\widehat{\theta}_{0}(\nu ; t)=\frac{1}{\sqrt{\pi x}}\left\{\sum_{n=0}^{\infty} \exp \left(-\frac{1}{x}\left(\nu+n+\frac{1}{2}\right)^{2}\right)-\sum_{n=-1}^{-\infty} n \cdot \exp \left(-\frac{1}{x}\left(\nu+n+\frac{1}{2}\right)^{2}\right)\right\}
$$

is the modified theta function, and

$$
\begin{gathered}
-\left[\frac{\partial}{\partial \nu} \widehat{\theta}_{0}\left(\frac{\nu}{2} ; x\right)\right]_{\nu=0}= \\
=\frac{1}{2 \sqrt{\pi} x^{\frac{3}{2}}}\left\{\sum_{n=0}^{+\infty}(2 n+1) \exp \left(-\frac{(2 n+1)^{2}}{4 x}\right)-\sum_{n=-1}^{-\infty}(2 n+1) \exp \left(-\frac{(2 n+1)^{2}}{4 x}\right)\right\}= \\
=\frac{1}{\sqrt{\pi} x^{\frac{3}{2}}} \sum_{n=0}^{\infty}(2 n+1) \exp \left(-\frac{(2 n+1)^{2}}{4 x}\right) .
\end{gathered}
$$

A particular solution of the differential equation (6) is the function:

$$
\bar{y}_{\text {part }}(p)=-\frac{1}{2 \operatorname{sh} \frac{\sqrt{p}}{a}} \int_{p}^{\infty} \bar{G}_{1}(q) \exp \left(\frac{\sqrt{q}}{a}\right) d q
$$

where

$$
\bar{G}_{1}(q)=\int_{0}^{\infty} e^{-q t} g_{1}(t) d t
$$

By virtue of replacements:

$$
t=\frac{1}{t_{1}}, \quad \tau=\frac{1}{\tau_{1}}
$$

and designations:

$$
y\left(t_{1}\right)=\frac{1}{t_{1}^{3 / 2}} \psi\left(\frac{1}{t_{1}}\right)
$$


from (9), we obtain the solution of the homogeneous equation corresponding to the integral equation (4):

$$
\psi_{\text {hom }}(t)=\frac{C}{a^{3} \sqrt{\pi}} \sum_{n=0}^{\infty}(2 n+1) \exp \left(-\frac{(2 n+1)^{2}}{4 a^{2}} t\right) .
$$

Then, the solution of the homogeneous equation, corresponding to the original an integral equation (1), has the form:

$$
\psi_{\text {hom }}(t)=\frac{C}{a^{3} \sqrt{\pi}} \sum_{n=0}^{\infty}(2 n+1) \exp \left(-\frac{n^{2}+n}{a^{2}} t\right) .
$$

The following theorem is proved:

Theorem 1. The integral equation

$$
\begin{aligned}
& \psi(t)-\frac{1}{2 a \sqrt{\pi}} \int_{t}^{\infty}\left[\frac{\tau+t}{(\tau-t)^{\frac{3}{2}}} \exp \left\{-\frac{(\tau+t)^{2}}{4 a^{2}(\tau-t)}\right\}+\right. \\
& \left.+\frac{1}{(\tau-t)^{\frac{1}{2}}} \exp \left\{-\frac{\tau-t}{4 a^{2}}\right\}\right] \psi(\tau) d \tau=0, \quad(t>0)
\end{aligned}
$$

in the class of essentially bounded functions at $t \geq t_{0}>0$ has the solution

$$
\psi(t)=\frac{C}{a^{3} \sqrt{\pi}} \sum_{n=0}^{\infty}(2 n+1) \exp \left(-\frac{n^{2}+n}{a^{2}} t\right)
$$

moreover, the norm of an integral operator acting in classes of continuous functions is equal to 3.

\section{Solving the nonhomogeneous equation with a difference kernel}

Next, we proceed to solving the corresponding nonhomogeneous equation.

As:

$$
\bar{G}_{1}(q)=\int_{0}^{\infty} e^{-q t} g_{1}(t) d t
$$

then (10) can be rewritten as

$$
\begin{gathered}
\bar{y}_{\text {part }}(p)=-\frac{1}{2 \operatorname{sh} \frac{\sqrt{p}}{a}} \int_{p}^{\infty} \exp \left(\frac{\sqrt{q}}{a}\right) d q \int_{0}^{\infty} e^{-q t_{2}} g_{1}\left(t_{2}\right) d t_{2}= \\
=-\frac{1}{2 \operatorname{sh} \frac{\sqrt{p}}{a}} \int_{0}^{\infty} g_{1}\left(t_{2}\right) d t_{2} \int_{p}^{\infty} \exp \left(-q t_{2}+\frac{\sqrt{q}}{a}\right) d q .
\end{gathered}
$$

In (12) we calculate the inner integral:

$$
\begin{gathered}
\int_{p}^{\infty} \exp \left(-q t_{2}+\frac{\sqrt{q}}{a}\right) d q=\left\|\frac{\sqrt{q}}{a}=z ; \quad q=a^{2} z^{2} ; \quad d q=2 a^{2} z d z\right\|= \\
=2 a^{2} \int_{\frac{\sqrt{p}}{a}}^{\infty} z \cdot \exp \left(-t_{2} a^{2} z^{2}+z\right) d z=\left\|\xi=a \sqrt{t_{2}} z-\frac{1}{2 a \sqrt{t_{2}}}\right\|= \\
=2 a^{2}\left[\frac{1}{a^{2} t_{2}} \exp \left(\frac{1}{4 a^{2} t_{2}}\right)\left\{\frac{1}{2 a \sqrt{t_{2}}} \int_{\sqrt{t_{2} p}-\frac{1}{2 a \sqrt{t_{2}}}}^{\infty} e^{-\xi^{2}} d \xi+\int_{\sqrt{t_{2} p}-\frac{1}{2 a \sqrt{t_{2}}}}^{\infty} \xi \cdot e^{-\xi^{2}} d \xi\right\}=\right. \\
=\frac{\sqrt{\pi}}{2 a t^{\frac{3}{2}}} \exp \left(\frac{1}{4 a^{2} t_{2}}\right) \cdot \operatorname{erfc}\left(\sqrt{t_{2}} \sqrt{p}-\frac{1}{2 a \sqrt{t_{2}}}\right)+\frac{1}{t_{2}} \exp \left(\frac{1}{4 a^{2} t_{2}}\right) \cdot \exp \left\{-\left(\sqrt{t_{2}} \sqrt{p}-\frac{1}{2 a \sqrt{t_{2}}}\right)^{2}\right\} .
\end{gathered}
$$


We introduce the notation:

$$
\begin{gathered}
\widehat{A}\left(t_{2} ; p\right)=\frac{\sqrt{\pi}}{2 a t_{2}^{\frac{3}{2}}} \exp \left(\frac{1}{4 a^{2} t_{2}}\right) \cdot \operatorname{erfc}\left(\sqrt{t_{2}} \sqrt{p}-\frac{1}{2 a \sqrt{t_{2}}}\right)+ \\
+\frac{1}{t_{2}} \exp \left(\frac{1}{4 a^{2} t_{2}}\right) \cdot \exp \left\{-\left(\sqrt{t_{2}} \sqrt{p}-\frac{1}{2 a \sqrt{t_{2}}}\right)^{2}\right\} .
\end{gathered}
$$

Then, taking into account the notation (13), the function (12) takes the form:

$$
\begin{gathered}
\bar{y}_{\text {part }}(p)=-\frac{1}{2 \operatorname{sh} \frac{\sqrt{p}}{a}} \int_{0}^{\infty} \widehat{A}\left(t_{2} ; p\right) g_{1}\left(t_{2}\right) d t_{2}=-\int_{0}^{\infty} \widehat{G}\left(t_{2} ; p\right) g_{1}\left(t_{2}\right) d t_{2}= \\
=-\int_{0}^{\infty}\left(\widehat{G}_{1}\left(t_{2} ; \sqrt{p}\right)+\widehat{G}_{1}\left(t_{2} ; \sqrt{p}\right)\right) g_{1}\left(t_{2}\right) d t_{2},
\end{gathered}
$$

where

$$
\widehat{G}\left(t_{2} ; p\right)=\widehat{G}_{1}\left(t_{2} ; \sqrt{p}\right)+\widehat{G}_{1}\left(t_{2} ; \sqrt{p}\right)
$$

and

$$
\begin{gathered}
\widehat{G}_{1}\left(t_{2} ; \sqrt{p}\right)=\frac{\sqrt{\pi}}{4 a t_{2}^{\frac{3}{2}}} \operatorname{sh} \frac{\sqrt{p}}{a} \exp \left(\frac{1}{4 a^{2} t_{2}}\right) \cdot \operatorname{erfc}\left(\sqrt{t_{2}} \sqrt{p}-\frac{1}{2 a \sqrt{t_{2}}}\right) \\
\widehat{G}_{2}\left(t_{2} ; \sqrt{p}\right)=\frac{1}{2 t_{2} \operatorname{sh} \frac{\sqrt{p}}{a}} \exp \left(\frac{1}{4 a^{2} t_{2}}\right) \cdot \exp \left\{-\left(\sqrt{t_{2}} \sqrt{p}-\frac{1}{2 a \sqrt{t_{2}}}\right)^{2}\right\} .
\end{gathered}
$$

We consider the latest equalities:

$$
\begin{gathered}
\widehat{G}_{1}\left(t_{2} ; \sqrt{p}\right)=\frac{\sqrt{\pi}}{2 a t_{2}^{\frac{3}{2}}}\left(e^{\frac{\sqrt{p}}{a}}-e^{-\frac{\sqrt{p}}{a}}\right) \exp \left(\frac{1}{4 a^{2} t_{2}}\right) \cdot \operatorname{erfc}\left(\sqrt{t_{2}} \sqrt{p}-\frac{1}{2 a \sqrt{t_{2}}}\right)= \\
=\frac{\sqrt{\pi}}{2 a t_{2}^{\frac{3}{2}}} \cdot e^{\frac{1}{4 a^{2} t}} \cdot e^{-\frac{\sqrt{p}}{a}+t_{2} p} \cdot \operatorname{erfc}\left(\sqrt{t_{2}} \cdot \sqrt{p}-\frac{1}{2 a \sqrt{t_{2}}}\right) \cdot e^{-t_{2} p} \cdot \frac{1}{1-e^{-\frac{2 \sqrt{p}}{a}}}= \\
=\frac{1}{2} \widehat{G}_{1}^{(1)}\left(t_{2}, \sqrt{p}\right) \cdot e^{-t_{2} p} \cdot \frac{1}{1-e^{-\frac{2 \sqrt{p}}{a}}} \cdot
\end{gathered}
$$

If in expression

$$
\widetilde{G}_{1}^{(1)}\left(t_{2}, \sqrt{p}\right)=\frac{\sqrt{\pi}}{a t_{2}^{\frac{3}{2}}} \cdot e^{\frac{1}{4 a^{2} t}} \cdot e^{-\frac{\sqrt{p}}{a}+t_{2} p} \cdot \operatorname{erfc}\left(\sqrt{t_{2} \cdot p}-\frac{1}{2 a \sqrt{t_{2}}}\right)
$$

from (16) to replace $\sqrt{p}$ with $p$, then from it is known that

$$
\widehat{G}_{1}^{(1)}\left(t_{2}, p\right) \bullet=\frac{1}{a t_{2}^{2}} \exp \left\{-\frac{t_{1}^{2}}{4 t_{2}}+\frac{t_{1}}{2 a t_{2}}\right\} .
$$

To find $\hat{G}_{1}\left(t_{2}, \sqrt{p}\right)$, we use the relation considering that $\hat{F}(p) \bullet={ }^{\bullet}(\tau)$ :

$$
\widehat{F}(\sqrt{p}) \bullet=\cdot \frac{1}{2 \sqrt{\pi} t_{1}^{\frac{3}{2}}} \int_{0}^{\infty} \tau \exp \left\{-\frac{\tau^{2}}{4 t_{1}}\right\} f(\tau) d \tau
$$

Then from (17) we get:

$$
\widetilde{G}_{1}^{(1)}\left(t_{2}, \sqrt{p}\right) \bullet=\cdot G_{1}^{(1)}\left(t_{2}, t_{1}\right) .
$$

Taking into account (18), the function-original (19) can be rewritten as:

$$
G_{1}^{(1)}\left(t_{2}, t_{1}\right)=\frac{1}{2 \sqrt{\pi} t_{1}^{\frac{3}{2}}} \int_{0}^{\infty} \tau \exp \left\{-\frac{\tau^{2}}{4 t_{1}}\right\} \frac{1}{a t_{2}^{2}} \exp \left\{-\frac{\tau^{2}}{4 t_{2}}+\frac{\tau}{2 a t_{2}}\right\} d \tau=
$$




$$
\begin{gathered}
=\frac{1}{2 a \sqrt{\pi}} \frac{1}{t_{1}^{\frac{3}{2}}} \cdot \frac{1}{t_{2}^{2}} \int_{0}^{\infty} \tau \exp \left\{-\tau^{2} \frac{t_{1}+t_{2}}{4 t_{1} t_{2}}+\tau \frac{1}{2 a t_{2}}\right\} d \tau=\frac{1}{2 a \sqrt{\pi} t_{2}^{2}} \frac{1}{t_{1}^{\frac{3}{2}}} \times \\
\times \frac{2 t_{1} t_{2}}{\left(t_{1}+t_{2}\right)} \exp \left\{\frac{t_{1} t_{2}}{4 a^{2} t_{2}^{2} 2\left(t_{1}+t_{2}\right)}\right\} D_{-2}\left(-\frac{\sqrt{2 t_{1} t_{2}}}{2 a t_{2} \sqrt{t_{1}+t_{2}}}\right)= \\
=\frac{1}{a \sqrt{\pi} t_{2} \sqrt{t_{1}}\left(t_{1}+t_{2}\right)}+\frac{1}{2 a^{2} t_{2}^{\frac{3}{2}}\left(t_{1}+t_{2}\right)^{\frac{3}{2}}} \exp \left\{\frac{t_{1}}{8 a^{2} t_{2}\left(t_{1}+t_{2}\right)}\right\} \operatorname{erfc}\left(-\frac{\sqrt{t_{1}}}{2 a \sqrt{t_{2}} \sqrt{t_{1}+t_{2}}}\right) .
\end{gathered}
$$

Let's go back to the relationship (16):

$$
\widehat{G}_{1}\left(t_{2}, \sqrt{p}\right)=\frac{1}{2} \widehat{G}_{1}^{(1)}\left(t_{2}, \sqrt{p}\right) \cdot e^{-t_{2} \cdot p} \frac{1}{1-e^{-\frac{2 \sqrt{p}}{a}}} .
$$

We use the following property of the Laplace transform (Time shifting):

$$
e^{-\alpha p} F(p) \bullet=\bullet f(t-\alpha),
$$

here $F(p) \bullet \cdot \bullet f(t)$.

From here

$$
\hat{G}_{1}^{(1)}\left(t_{2}, p\right) \cdot e^{-t_{2} p} \bullet \cdot G_{1}^{(1)}\left(t_{2}, t_{1}-t_{2}\right) .
$$

We write the expression $G_{1}^{(1)}\left(t_{2}, t_{1}-t_{2}\right)$ from (22) explicitly using the formula (20):

$$
G_{1}^{(1)}\left(t_{2}, t_{1}-t_{2}\right)=\frac{1}{a \sqrt{\pi} t_{2} \sqrt{t_{1}-t_{2}} t_{1}}+\frac{\exp \left(\frac{t_{1}-t_{2}}{8 a^{2} t_{2} t_{1}}\right)}{2 a^{2} t_{1}^{\frac{3}{2}} t_{2}^{\frac{3}{2}}} \operatorname{erfc}\left(\frac{-\sqrt{t_{1}-t_{2}}}{2 a \sqrt{t_{2}} \sqrt{t_{1}}}\right) .
$$

It should be noted that $G_{1}^{(1)}\left(t_{2}, t_{1}-t_{2}\right) \neq 0$ when $t_{1}>t_{2}$.

Next we will find the original of the last factor (image) in the ratio (21):

$$
\frac{1}{1-e^{-\frac{2 \sqrt{p}}{a}}}=1+\sum_{n=1}^{\infty} \exp \left(-\frac{2 n}{a} \sqrt{p}\right) \cdot=^{\bullet} \delta\left(t_{1}\right)+\frac{1}{a \sqrt{\pi} t^{\frac{3}{2}}} \sum_{n=1}^{\infty} n \cdot \exp \left(-n^{2} \cdot \frac{1}{a^{2} t_{1}}\right) .
$$

Using the convolution theorem, relations (23) and (24) we obtain:

$$
\begin{gathered}
G_{1}\left(t_{2}, t_{1}\right)=\frac{1}{2} \int_{0}^{t_{1}-t_{2}} G_{1}^{(1)}\left(t_{2}, t_{1}-t_{2}-\tau\right) \cdot\left[\delta(\tau)+\frac{1}{a \sqrt{\pi} \tau^{\frac{3}{2}}} \sum_{n=1}^{\infty} n \cdot \exp \left(-n^{2} \cdot \frac{1}{a^{2} t_{1}}\right)\right] d \tau= \\
=\frac{1}{2} \int_{t_{2}}^{t_{1}} G_{1}^{(1)}\left(t_{2}, \tau-t_{2}\right)\left[\delta\left(t_{1}-\tau\right)+\frac{1}{a \sqrt{\pi}} \sum_{n=1}^{\infty} n \cdot \exp \left(-n^{2} \frac{1}{a^{2}\left(t_{1}-\tau\right)}\right)\right] d \tau= \\
=\frac{1}{2} G_{1}^{(1)}\left(t_{2}, t_{1}-t_{2}\right)+\frac{1}{2 a \sqrt{\pi}} \sum_{n=1}^{\infty} \int_{t_{2}}^{t_{1}} n \cdot \exp \left(-n^{2} \frac{1}{a^{2}\left(t_{1}-\tau\right)}\right) \cdot G_{1}^{(1)}\left(t_{2}, \tau-t_{2}\right) d \tau .
\end{gathered}
$$

Then the original of the obtained solution (14) has the form:

$$
y_{\text {part }}\left(t_{1}\right)=-\int_{0}^{t_{1}} G\left(t_{2}, t_{1}\right) g_{1}\left(t_{2}\right) d t_{2}=-\left(\int_{0}^{t_{1}} G_{1}\left(t_{2}, t_{1}\right) g_{1}\left(t_{2}\right) d t_{2}+\int_{0}^{t_{1}} G_{2}\left(t_{2}, t_{1}\right) g_{1}\left(t_{2}\right) d t_{2}\right),
$$

where

$$
G\left(t_{2}, t_{1}\right)=G_{1}\left(t_{2}, t_{1}\right)+G_{2}\left(t_{2}, t_{1}\right)
$$

is the original of the function (15).

Now we find $G_{2}\left(t_{2}, t_{1}\right)$.

$$
\widehat{G}_{2}\left(t_{2} ; \sqrt{p}\right)=\frac{1}{2 t_{2} \operatorname{sh} \frac{\sqrt{p}}{0}} \exp \left(\frac{1}{4 a^{2} t_{2}}\right) \cdot \exp \left\{-\left(\sqrt{t_{2}} \sqrt{p}-\frac{1}{2 a \sqrt{t_{2}}}\right)^{2}\right\}=
$$




$$
=\frac{1}{e^{\frac{\sqrt{p}}{a}}-e^{-\frac{\sqrt{p}}{a}}} \cdot \frac{1}{t_{2}} \cdot \exp \left(\frac{1}{4 a^{2} t_{2}}-t_{2} p+\frac{\sqrt{p}}{a}-\frac{1}{4 a^{2} t_{2}}\right)=\frac{1}{t_{2}} e^{-t_{2} p} \cdot \frac{1}{1-e^{-\frac{2 \sqrt{p}}{a}}} .
$$

Then we have:

$$
\begin{gathered}
\widehat{G}_{2}\left(t_{2}, p\right)=\frac{1}{t_{2}} \sum_{n=0}^{\infty} e^{-t_{2} p} \cdot e^{-\frac{2 n \sqrt{p}}{a}}=\frac{1}{t_{2}} e^{-t_{2} p}\left\{1+\sum_{n=1}^{\infty} e^{-\frac{2 n \sqrt{p}}{a}}\right\} \cdot=\cdot \\
\bullet=\cdot \frac{1}{t_{2}}\left\{\delta\left(t_{1}-t_{2}\right)+\sum_{n=1}^{\infty} n \cdot \frac{e^{-\frac{n^{2}}{a^{2}\left(t_{1}-t_{2}\right)}}}{a \sqrt{\pi}\left(t_{1}-t_{2}\right)^{\frac{3}{2}}}\right\}=G_{2}\left(t_{2}, t_{1}\right) .
\end{gathered}
$$

and $\frac{1}{t_{2}}\left\{\delta\left(t_{1}-t_{2}\right)+\sum_{n=1}^{\infty} n \cdot \frac{e^{-\frac{n^{2}}{a^{2}\left(t_{1}-t_{2}\right)}}}{a \sqrt{\pi}\left(t_{1}-t_{2}\right)^{\frac{3}{2}}}\right\}=0 ;$ at $t_{2}>t_{1}$.

Then the second term in the solution (26) takes the form:

$$
\begin{gathered}
y_{\text {part }}^{(2)}\left(t_{1}\right)=-\int_{0}^{t_{1}}\left\{\delta\left(t_{1}-t_{2}\right)+\sum_{n=1}^{\infty} n \cdot \frac{e^{-\frac{n^{2}}{a^{2}\left(t_{1}-t_{2}\right)}}}{a \sqrt{\pi}\left(t_{1}-t_{2}\right)^{\frac{3}{2}}}\right\} \frac{1}{t_{2}} g_{1}\left(t_{2}\right) d t_{2}= \\
=-\frac{1}{t_{1}} g_{1}\left(t_{1}\right)-\frac{1}{a \sqrt{\pi}} \sum_{n=1}^{\infty} \int_{0}^{t_{1}} \frac{n}{\left(t_{1}-t_{2}\right)^{\frac{3}{2}}} \cdot e^{-\frac{n^{2}}{a^{2}\left(t_{1}-t_{2}\right)}} \cdot \frac{1}{t_{2}} \cdot g_{1}\left(t_{2}\right) d t_{2} .
\end{gathered}
$$

Finally taking into account (25) and (26) we have:

$$
\begin{gathered}
y_{\text {part }}\left(t_{1}\right)=-\frac{1}{t_{1}} g_{1}\left(t_{1}\right)-\frac{1}{a \sqrt{\pi}} \sum_{n=1}^{\infty} \int_{0}^{t_{1}} \frac{n}{\left(t_{1}-t_{2}\right)^{\frac{3}{2}} t_{2}} \exp \left(-\frac{n^{2}}{a^{2}\left(t_{1}-t_{2}\right)}\right) g_{1}\left(t_{2}\right) d t_{2}- \\
-\frac{1}{2} \int_{0}^{t_{1}} G_{1}\left(t_{2}, t_{1}\right) g_{1}\left(t_{2}\right) d t_{2} .
\end{gathered}
$$

Then the solution of equation (5) has the form:

$$
y\left(t_{1}\right)=y_{\text {hom }}\left(t_{1}\right)+y_{\text {part }}\left(t_{1}\right)
$$

where $y_{\text {part }}\left(t_{1}\right)$ is determined by formula (27) and the solution of the corresponding homogeneous equation was determined above:

$$
y_{\text {hom }}\left(t_{1}\right)=-C\left[\frac{\partial}{\partial \nu} \widehat{\theta}_{0}\left(\frac{\nu}{2} ; a^{2} t_{1}\right)\right]_{\nu=0}
$$

and

$$
-\left[\frac{\partial}{\partial \nu} \widehat{\theta}_{0}\left(\frac{\nu}{2} ; x\right)\right]_{\nu=0}=\frac{1}{\sqrt{\pi} x^{\frac{3}{2}}} \sum_{n=0}^{\infty}(2 n+1) \exp \left(-\frac{(2 n+1)^{2}}{4 x}\right) .
$$

Let's go back to the old variables.

Earlier, the replacement $t=\frac{1}{t_{1}}$ and the following designations were introduced:

$$
y\left(t_{1}\right)=\frac{1}{t_{1}^{\frac{3}{2}}} \psi\left(\frac{1}{t_{1}}\right), \quad g_{1}\left(t_{1}\right)=\frac{1}{\sqrt{t_{1}}} g\left(\frac{1}{t_{1}}\right) .
$$

Therefore, from (23) we have (here: $t_{2}=\frac{1}{\tau}, \quad t_{1}=\frac{1}{t}, \quad \tau=\frac{1}{\tau_{1}}$ ):

$$
G_{1}^{(1)}\left(t_{2}, t_{1}-t_{2}\right)=G_{1}^{(1)}\left(\frac{1}{\tau}, \frac{1}{t}-\frac{1}{\tau}\right)=\frac{t^{\frac{3}{2}} \tau^{\frac{3}{2}}}{a \sqrt{\pi} \sqrt{\tau-t}}+\frac{t^{\frac{3}{2}} \tau^{\frac{3}{2}} \exp \left(\frac{\tau-t}{8 a^{2}}\right)}{2 a^{2}} \operatorname{erfc}\left(\frac{-\sqrt{\tau-t}}{2 a}\right) .
$$


From the last formula for (25) we get:

$$
\begin{gathered}
G_{1}\left(t_{2}, t_{1}\right)=G_{1}\left(\frac{1}{\tau}, \frac{1}{t}\right)=\frac{t^{\frac{3}{2}} \tau^{\frac{3}{2}}}{2 a \sqrt{\pi} \sqrt{\tau-t}}+\frac{t^{\frac{3}{2}} \tau^{\frac{3}{2}} \exp \left(\frac{\tau-t}{8 a^{2}}\right)}{4 a^{2}} \operatorname{erfc}\left(\frac{-\sqrt{\tau-t}}{2 a}\right)+ \\
+\frac{1}{2 a^{2} \sqrt{\pi}} \sum_{n=1}^{\infty} \int_{\tau}^{t} n \cdot \exp \left(-n^{2} \frac{\tau_{1} t}{a^{2}\left(\tau_{1}-t\right)}\right) \times \\
\times\left(\frac{\tau_{1}^{\frac{3}{2}} \tau^{\frac{3}{2}}}{\sqrt{\pi} \sqrt{\tau-\tau_{1}}}+\frac{\tau_{1}^{\frac{3}{2}} \tau^{\frac{3}{2}} \exp \left(\frac{\tau-\tau_{1}}{8 a^{2}}\right)}{2 a} \operatorname{erfc}\left(\frac{-\sqrt{\tau-\tau_{1}}}{2 a}\right)\right) \frac{d \tau_{1}}{\tau_{1}^{2}} .
\end{gathered}
$$

Then the solution (27) can be rewritten in the form:

$$
\begin{gathered}
y_{\text {part }}\left(t_{1}\right)=-\frac{1}{t_{1}} g_{1}\left(t_{1}\right)-\frac{1}{a \sqrt{\pi}} \sum_{n=1}^{\infty} \int_{0}^{t_{1}} \frac{n}{\left(t_{1}-t_{2}\right)^{\frac{3}{2}} t_{2}} \exp \left(-\frac{n^{2}}{a^{2}\left(t_{1}-t_{2}\right)}\right) g_{1}\left(t_{2}\right) d t_{2}- \\
\quad-\frac{1}{2} \int_{0}^{t_{1}} G_{1}\left(t_{2}, t_{1}\right) \cdot g_{1}\left(t_{2}\right) d t_{2} \\
t^{\frac{3}{2}} \psi_{\text {part }}(t)=-t^{\frac{3}{2}} g(t)-\frac{1}{a \sqrt{\pi}} \sum_{n=1}^{\infty} \int_{t}^{\infty} \frac{\tau \cdot n}{\left(\frac{1}{t}-\frac{1}{\tau}\right)^{\frac{3}{2}}} \exp \left(-\frac{n^{2}}{a^{2}\left(\frac{1}{t}-\frac{1}{\tau}\right)}\right) \sqrt{\tau} g(\tau) \frac{d \tau}{\tau^{2}}- \\
-\frac{1}{2} \int_{t}^{\infty} \frac{t^{\frac{3}{2}} \tau^{\frac{3}{2}}}{2 a \sqrt{\pi} \sqrt{\tau-t}} \cdot \sqrt{\tau} g(\tau) \frac{d \tau}{\tau^{2}}-\frac{1}{2} \int_{t}^{\infty} \frac{t^{\frac{3}{2}} \tau^{\frac{3}{2}} \exp \left(\frac{\tau-t}{8 a^{2}}\right)}{4 a^{2}} \operatorname{erfc}\left(\frac{-\sqrt{\tau-t}}{2 a}\right) \cdot \sqrt{\tau} g(\tau) \frac{d \tau}{\tau^{2}}- \\
-\frac{1}{2} \int_{t}^{\infty} \frac{1}{2 a^{2} \sqrt{\pi}} \sum_{n=1}^{\infty} \int_{\tau}^{t} n \exp \left(-n^{2} \frac{\tau_{1} t}{a^{2}\left(\tau_{1}-t\right)}\right)\left(\frac{\tau_{1}^{\frac{3}{2}} \tau^{\frac{3}{2}}}{\sqrt{\pi} \sqrt{\tau-\tau_{1}}}+\right. \\
\left.+\frac{\tau_{1}^{\frac{3}{2}} \tau^{\frac{3}{2}} \exp \left(\frac{\tau-\tau_{1}}{8 a^{2}}\right)}{2 a} \operatorname{erfc}\left(\frac{-\sqrt{\tau-\tau_{1}}}{2 a}\right)\right) \frac{d \tau_{1}}{\tau_{1}^{2}} \sqrt{\tau} g(\tau) \frac{d \tau}{\tau^{2}} .
\end{gathered}
$$

After simplifications we get:

$$
\begin{gathered}
\psi_{\text {part }}(t)=-g(t)-\frac{1}{a \sqrt{\pi}} \sum_{n=1}^{\infty} \int_{t}^{\infty} \frac{\tau n}{(\tau-t)^{\frac{3}{2}}} \cdot \exp \left(-\frac{n^{2} t \tau}{a^{2}(\tau-t)}\right) g(\tau) d \tau- \\
-\frac{1}{4 a \sqrt{\pi}} \int_{t}^{\infty} \frac{1}{\sqrt{\tau-t}} g(\tau) d \tau-\frac{1}{8 a^{2}} \int_{t}^{\infty} \exp \left(\frac{\tau-t}{8 a^{2}}\right) \operatorname{erfc}\left(\frac{-\sqrt{\tau-t}}{2 a}\right) \cdot g(\tau) d \tau- \\
-\frac{1}{4 a^{2} \sqrt{\pi} t^{\frac{3}{2}}} \int_{t}^{\infty} \sum_{n=1}^{\infty} \int_{\tau}^{t} \frac{n}{\sqrt{\tau_{1}}} \exp \left(-n^{2} \frac{\tau_{1} t}{a^{2}\left(\tau_{1}-t\right)}\right)\left(\frac{1}{\sqrt{\pi} \sqrt{\tau-\tau_{1}}}+\right. \\
\left.+\frac{\exp \left(\frac{\tau-\tau_{1}}{8 a^{2}}\right)}{2 a \sqrt{\tau_{1}}} \operatorname{erfc}\left(\frac{-\sqrt{\tau-\tau_{1}}}{2 a}\right)\right) d \tau_{1} g(\tau) d \tau
\end{gathered}
$$

or

$$
\begin{gathered}
\psi_{\text {part }}(t)=-g(t)-\frac{1}{a \sqrt{\pi}} \sum_{n=1}^{\infty} \int_{t}^{\infty} \frac{\tau \cdot n}{(\tau-t)^{\frac{3}{2}}} \exp \left(-\frac{n^{2} t \tau}{a^{2}(\tau-t)}\right) g(\tau) d \tau- \\
-\frac{1}{4 a \sqrt{\pi}} \int_{t}^{\infty} \frac{1}{\sqrt{\tau-t}} g(\tau) d \tau-\frac{1}{8 a^{2}} \int_{t}^{\infty} \exp \left(\frac{\tau-t}{8 a^{2}}\right) \operatorname{erfc}\left(\frac{-\sqrt{\tau-t}}{2 a}\right) \cdot g(\tau) d \tau-
\end{gathered}
$$




$$
\begin{gathered}
-\frac{1}{4 a^{2} \sqrt{\pi} t^{\frac{3}{2}}} \int_{t}^{\infty} g(\tau) \int_{\tau}^{t}\left(\frac{1}{\sqrt{\pi} \sqrt{\tau_{1}} \sqrt{\tau-\tau_{1}}}+\frac{1}{2 a \tau_{1}} \exp \left(\frac{\tau-\tau_{1}}{8 a^{2}}\right) \operatorname{erfc}\left(\frac{-\sqrt{\tau-\tau_{1}}}{2 a}\right)\right) \times \\
\times \sum_{n=1}^{\infty} n \cdot \exp \left(-n^{2} \frac{\tau_{1} t}{a^{2}\left(\tau_{1}-t\right)}\right) d \tau_{1} \cdot d \tau .
\end{gathered}
$$

Then the solution of the integral equation (3) taking into account the obtained expressions (29) or (11) (see Theorem 1) and (30) has the explicit form:

$$
\begin{gathered}
\psi(t)=\frac{C}{a^{3} \sqrt{\pi}} \sum_{n=0}^{\infty}(2 n+1) \exp \left(-\frac{n^{2}+n}{a^{2}} t\right)-g(t)- \\
-\frac{1}{a \sqrt{\pi}} \sum_{n=1}^{\infty} \int_{t}^{\infty} \frac{\tau n}{(\tau-t)^{\frac{3}{2}}} \exp \left(-\frac{n^{2} t \tau}{a^{2}(\tau-t)}\right) \cdot g(\tau) d \tau- \\
-\frac{1}{4 a \sqrt{\pi}} \int_{t}^{\infty} \frac{1}{\sqrt{\tau-t}} g(\tau) d \tau-\frac{1}{8 a^{2}} \int_{t}^{\infty} \exp \left(\frac{\tau-t}{8 a^{2}}\right) \operatorname{erfc}\left(\frac{-\sqrt{\tau-t}}{2 a}\right) \cdot g(\tau) d \tau- \\
\quad \frac{1}{4 a^{2} \sqrt{\pi} t^{\frac{3}{2}}} \int_{t}^{\infty} g(\tau) \int_{\tau}^{t}\left(\frac{1}{\sqrt{\pi} \sqrt{\tau_{1}} \sqrt{\tau-\tau_{1}}}+\frac{1}{2 a \tau_{1}} \exp \left(\frac{\tau-\tau_{1}}{8 a^{2}}\right) \operatorname{erfc}\left(\frac{-\sqrt{\tau-\tau_{1}}}{2 a}\right)\right) \times \\
\times \sum_{n=1}^{\infty} n \exp \left(-n^{2} \frac{\tau_{1} t}{a^{2}\left(\tau_{1}-t\right)}\right) d \tau_{1} d \tau .
\end{gathered}
$$

\section{Main results}

Theorem 2. The solution of the integral equation (1) with the singular kernel (2) has an explicit form defined by the formula (31).

Remark. Singular homogeneous integral equations were considered in works [1-4]. Their kernels were also incompressible, but kernels had an another form. In this connection, the weight classes of the solution existence differ from the class of the solution existence for the equation considered in this work. We also note that boundary value problems for a spectrally loaded parabolic equation reduce to this kind of singular integral equations, when the load line moves according to the law $x=t[5-10]$ and problems for essentially loaded equation of heat conduction [11-15].

In works $[16,17]$ it is shown that the homogeneous Volterra integral equation of the second kind, to which the homogeneous boundary value problem of heat conduction in the degenerating domain is reduced, has a nonzero solution.

In works $[18,19]$ boundary value problems for heat equation in angular domains with special boundary conditions are studied. The problems are reduced to singular integral equations of Volterra type of the second kind, similar to the equation (1).

\section{References}

1 Kosmakova, M.T., Akhmanova, D.M., Iskakov, S.A., Tuleutaeva, Zh.M., \& Kasymova, L.Zh. (2019). Solving one pseudo-Volterra integral equation. Bulletin of the Karaganda University. Mathematics series, 1 (93), 72-77. DOI: 10.31489/2019M1/72-77

2 Jenaliyev, M., Amangaliyeva, M., Kosmakova, M., \& Ramazanov, M. (2014). About Dirichlet boundary value problem for the heat equation in the infinite angular domain. Boundary Value Problems, 213, 1-21. DOI: $10.1186 / \mathrm{s} 13661-014-0213-4$

3 Amangaliyeva, M.M., Dzhenaliev, M.T., Kosmakova, M.T., \& Ramazanov M.I. (2015). On one homogeneous problem for the heat equation in an infinite angular domain. Siberian Mathematical Journal, Vol. 56, No. 6, 982-995. DOI: 10.1134/S0037446615060038

4 Jenaliyev, M., Amangaliyeva, M., Kosmakova, M., \& Ramazanov, M. (2015). On a Volterra equation of the second kind with «incompressible» kernel. Advances in Difference Equations, 71, 1-14. DOI: 10.1186/s13662-015-0418-6 
5 Akhmanova, D.M., Dzhenaliev, M.T., \& Ramazanov, M.I. (2011). On a particular second kind Volterra integral equation with a spectral parameter. Siberian Mathematical Journal, 52, 1, 1-10. DOI: $10.1134 /$ S0037446606010010

6 Amangaliyeva, M.M., Akhmanova, D.M., Dzhenaliev, M.T., \& Ramazanov, M.I. (2011). Boundary value problems for a spectrally loaded heat operator with load line approaching the time axis at zero or infinity. Differential Equations, 47, 2, 231-243. DOI: 10.1134/S0012266111020091

7 Kosmakova, M.T. (2016). On an integral equation of the Dirichlet problem for the heat equation in the degenerating domain. Bulletin of the Karaganda University. Mathematics series, 1 (81), 62-67.

8 Dzhenaliev, M.T., \& Ramazanov, M.I. (2006). On the boundary value problem for the spectrally loaded heat conduction operator. Siberian Mathematical Journal, 47, 3, 433-451. DOI: 10.1007/s11202-0060056-z

9 Dzhenaliev, M.T., \& Ramazanov, M.I. (2007). On a boundary value problem for a spectrally loaded heat operator: I. Differential Equations, 43, 4, 513-524. DOI: 10.1134/S0012266107040106

10 Dzhenaliev, M.T., \& Ramazanov, M.I. (2007). On a boundary value problem for a spectrally loaded heat operator: II. Differential Equations, 43, 6, 806-812. DOI: 10.1134/S0012266107060079

11 Ramazanov, M.I., Kosmakova, M.T., Romanovsky, V.G., Zhanbusinova, B.H., \& Tuleutaeva, Z.M. (2018). Boundary value problems for essentially-loaded parabolic equation. Bulletin of the Karaganda University. Mathematics series, 4 (92), 79-86. DOI: 10.31489/2018M4/79-86

12 Muvasharkhan Jenaliyev, M., \& Ramazanov, M. (2016). On a homogeneous parabolic problem in an infinite corner domain. AIP Conference Proceedings, 1759, 020085. DOI: 10.1063/1.4959699

13 Amangaliyeva, M.M., Jenaliyev, M.T., Kosmakova, M.T., \& Ramazanov, M.I. (2015). Uniqueness and non-uniqueness of solutions of the boundary value problems of the heat equation. AIP Conference Proceedings, 1676, 020028. DOI: 10.1063/1.4930454

14 Amangaliyeva, M.M., Jenaliyev, M.T., Kosmakova, M.T., \& Ramazanov, M.I. (2014). On the spectrum of Volterra integral equation with the «incompressible» kernel. AIP Conference Proceedings, 1611, 127-132. DOI: 10.1063/1.4893816

15 Kosmakova, M.T., Orumbayeva, N.T., Medeubaev, N.K., \& Tuleutaeva, Zh.M. (2018). Problems of Heat Conduction with Different Boundary Conditions in Noncylindrical Domains. AIP Conference Proceedings, 1997, UNSP 020071-1. DOI: 10.1063/1.5049065

16 Akhmanova, D.M., Ramazanov, M.I., \& Yergaliyev. M.G. (2018). On an integral equation of the problem of heat conduction with domain boundary moving by law of $\mathrm{t}=\mathrm{x}(2)$. Bulletin of the Karaganda University . Mathematics series, 1 (89), 15-19. DOI: 10.31489/2018M1/15-19

17 Kosmakova, M.T., Ramazanov, M.I., Tokesheva, A.S., \& Khairkulova, A.A. (2016). On the non-uniqueness of solution to the homogeneous boundary value problem for the heat conduction equation in an angular domain. Bulletin of the Karaganda University. Mathematics series, 4 (84), 80-87. DOI: 10.31489/2016M4 /80-87

18 Jenaliyev, M.T., Iskakov, S.A., \& Ramazanov, M.I. (2017). On a parabolic problem in an infinite corner domain. Bulletin of the Karaganda University. Mathematics series, 1 (85), 28-35. DOI: 10.31489/2017 $\mathrm{M} 1 / 28-35$

19 Jenaliyev, M.T., Iskakov, S.A., Ramazanov, M.I., \& Tuleutaeva, Z.M. (2018). On the solvability of the first boundary value problem for the loaded equation of heat conduction. Bulletin of the Karaganda University. Series Mathematics, 1 (89), 33-41.

\section{М.Т. Космакова, В.Г. Романовский, Н.Т. Орумбаева, Ж.М. Тулеутаева, Л.Ж. Касымова \\ Жылуөткізгіштіктің түйіндес есебінің бір интегралдық теңдеуі жайлы}

Жылуөткізгіштіктің түйіндес операторлы біртекті емес бірінші шеттік есебі келтірілетін интеграл-
дық теңдеу қарастырылды. Есеп шексіз жазық бұрышта қойылған, яғни облыстың шекарасы тұрақты 
жылдамдықпен қозғалады және облыс уақыттың бастапқы мезгілінде нүктеге айналады. Зерттелудегі теңдеудің интегралдық операторының сығылмайтыны көрсетілген. Тәуелсіз айнымалы үшін қатынастарды қолданып, зерттеліп отырған теңдеу қандайда бір эквивалентті ықшам теңдеуге келтірілді. Тәуелсіз айнымалылар үшін ауыстырулар көмегімен теңдеу айырымдық ядросы бар интегралдық теңдеуге келтірілді. Лаплас түрлендіруін қолдану арқылы алынған теңдеу қарапайым бірінші ретті дифференциалдық теңдеуге (сызықтық) келтірілді. Оның шешуі табылды. Лапластың кері түрленуінің көмегімен зерттелетін біртекті емес интегралды теңдеудің қандай да бір облыста жинақты қатар түріндегі шешуі алынды.

Kiлm сөздер: жылуөткізгіштік, біртекті емес сингулярлы интегралдық теңдеу, түйіндес шеттік есеп, Лаплас түрлендіруі.

\title{
М.Т. Космакова, В.Г. Романовский, Н.Т. Орумбаева, Ж.М. Тулеутаева, Л.Ж. Касымова \\ Об одном интегральном уравнении сопряженной задачи теплопроводности
}

\begin{abstract}
Рассмотрено интегральное уравнение, к которому сводится неоднородная первая краевая задача с сопряженным оператором теплопроводности. Задача поставлена в бесконечном плоском угле, т.е. граница области движется с постоянной скоростью, и область вырождается в точку в начальный момент времени. Показана несжимаемость интегрального оператора исследуемого уравнения. Используя соотношения для независимой переменной, исследуемое уравнение эквивалентно сводится к некоторому упрощенному уравнению. С помощью замен для независимых переменных уравнение сводится к интегральному уравнению с разностным ядром. Применением преобразования Лапласа полученное уравнение сведено к обыкновенному дифференциальному уравнению первого порядка (линейному). Найдено его решение. С помощью обратного преобразования Лапласа получено решение исследуемого неоднородного интегрального уравнения в виде сходящегося ряда в некоторой области.
\end{abstract}

Ключевые слова: теплопроводность, неоднородное сингулярное интегральное уравнение, сопряженная граничная задача, преобразование Лапласа. 